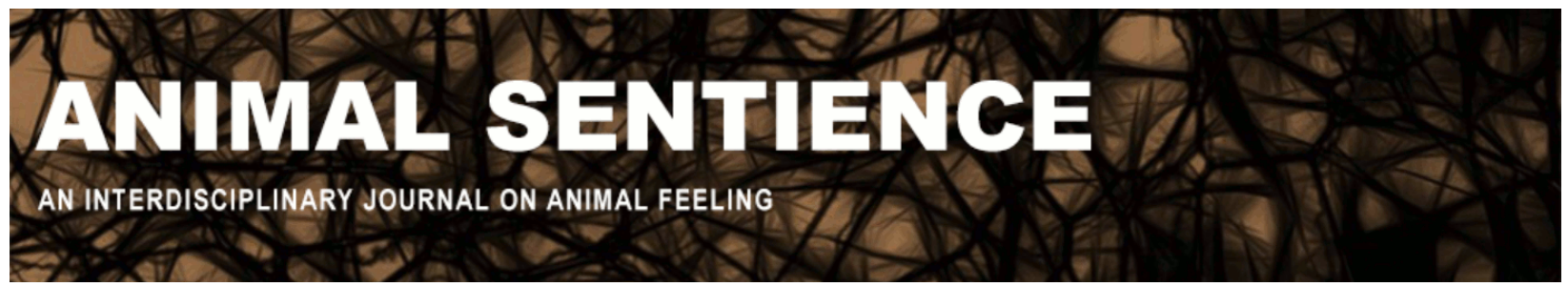

Adolphs, Ralph (2017) Dogs consciously experience emotions: The question is, which?. Animal Sentience 14(2)

DOI: $10.51291 / 2377-7478.1213$

Date of submission: 2017-10-01

Date of acceptance: 2017-10-03

(c)

This article has appeared in the journal Animal

Sentience, a peer-reviewed journal on animal

cognition and feeling. It has been made open access,

free for all, by WellBeing International and deposited

in the WBI Studies Repository. For more information,

please contact

wbisr-info@wellbeingintl.org.

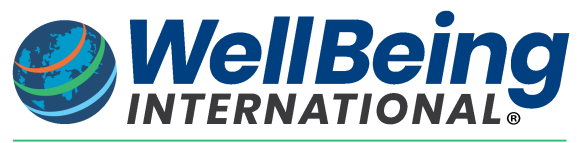

SOLUTIONS FOR PEOPLE, ANIMALS AND ENVIRONMENT 


\title{
Dogs consciously experience emotions: The question is, which?
}

Commentary on Kujala on Canine Emotions

\author{
Ralph Adolphs \\ California Institute of Technology
}

\begin{abstract}
I discuss three themes related to Kujala's target article. First, the wealth of emerging data on cognitive studies in dogs will surely show that dogs have a very rich repertoire of cognitive processes, for most of which we find homologues in humans. Second, understanding the internal states that mediate social behaviors, such as emotions, requires us to consider both a dog's behaviors with other dogs, and the emergence of new behavioral patterns in interaction with humans. Third, all of this will certainly narrow the range of justifications for denying that dogs have subjective experiences of emotions.
\end{abstract}

\begin{abstract}
Ralph Adolphs is a social neuroscientist who studies emotions in humans. Bren Professor of Psychology, Neuroscience and Biology at California Institute of Technology, Allen V. C. Davis and Lenabelle Davis Leadership Chair and Director at Caltech Brain Imaging Center, Adolphs is the co-author of The neuroscience of emotions in humans and animals (Princeton University Press, forthcoming, 2018). http://emotion.caltech.edu
\end{abstract}

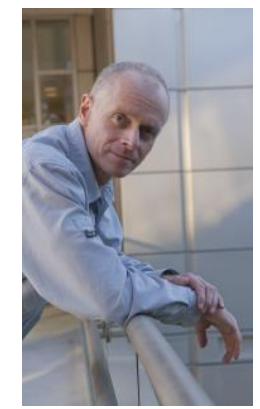

\section{A wealth of emerging data.}

Kujala (2017) reviews a large and rapidly growing literature, focused mostly on behavioral work but, more recently, also including neuroimaging and other data. Our own social perception and inference easily take a dog's behavior and let us infer a number of internal states, amongst them feelings, emotions, moods. But are we right?

As a new ingredient, Kujala briefly touches on neuroscience data, which is beginning to give us insight into what is happening in the dog's brain. Work by researchers such as Berns (2016) is giving us quite a bit of novel neuroimaging data about how the dog brain works. For the most part, it seems to work quite similarly to the brains of other higher mammals, including humans. The olfactory cortex is activated by odors (but, interestingly, only if the dog is not anesthetized). The smell of an owner (but not other people) activates reward-related brain regions (the ventral striatum). The secondary auditory cortex in dogs seems sensitive to the valence of a human voice (praise or punishment sounds). Somewhat similarly to primates and sheep, dogs seem to have visually responsive brain regions that respond disproportionately to faces. Beyond these perceptual aspects, higher cognitive processes such as behavioral inhibition and control have also been studied, and again seem to involve roughly the expected brain regions (e.g., lateral prefrontal cortex). 
It is important to keep in mind that there are the usual caveats in all this literature about reverse inference, and inference based on unreliable and sparse data. It is still early days for dog neuroimaging, but the data are accruing. (For some recent reviews, see the special issue on cognition in dogs, Current Directions in Psychological Science, Engle \& Zentall 2016). The results so far are beginning to paint a picture of a brain that shows many similarities to our own. All this, while far from definitive, is certainly consistent with the hypothesis that a dog brain implements many of the same psychological processes, or close homologues of them, as those found in a human brain.

\section{What emotions are there in dogs?}

Neuroethologists would tell us that the emotions that we should study in dogs are those that one might hypothesize in this species given its natural social behavior. Behaviors related to establishing dominance and submission in a social hierarchy would be amongst them. The problem is that we tend to apply inferences derived from our own species. Kujala touches on this in section 7: are dogs expressing guilt or appeasement? When you pet your dog, it rolls over on its back, exposes its belly and seems to be enjoying this. But what do other dogs do to one another? Rolling over and exposing your belly is a submissive behavior to establish dominance hierarchies. One possibility is that the similar behaviors in interactions with humans also signal dominance relations, not "happiness," and that we systematically misinterpret the internal states of dogs. Dogs do not whistle at, scold, praise, or pet one another, so whatever behaviors we elicit with these common interactions become ill-defined if we stick with interpreting them relative to the dog's conspecific environment.

But there is a second possibility: dogs have a repertoire of behaviors available that they can use more flexibly. Perhaps they are using the behaviors that they would use to signal dominance relationships amongst themselves for a different function when they interact with humans, perhaps a function closer to our "happiness." One very interesting question is whether, over time, dogs and other pets actually do acquire the emotions we anthropomorphize in them. Sure, they behave quite differently amongst one another than with a human owner. But, over time, the behavioral interactions may take on different meanings. Emotions may in fact be created through learning. Tameness may be a trait that enables such learning, which may be very difficult or impossible in most wild animals (who may also learn, but are fairly limited in learning only their species-specific interactions).

\section{Do dogs have conscious experiences?}

In my view, there are only two positions that would be consistent with denying that dogs consciously experience emotions. The first position is that all of the evidence is in principle conceivable in the absence of conscious feelings. Yes, it is conceivable that the conscious aspect of emotions serves no functional role and so is just disconnected from all of the behavioral and neural data; everything could be going on "in the dark." If you take this view, there is no reason not to take it also with humans, and you either deny conscious experiences altogether or believe they are "epiphenomenal." I assume that this is not an attractive nor a common view. 
The second view is that there is something functional missing that humans have and dogs don't. The two contenders are "metacognition" of some sort, and language. Dogs cannot tell us how they feel, and dogs may not have higher-order reflections on how they feel. This seems like a reasonable position, but it forces you to ground conscious experiences in language and/or metacognition. There is little evidence in humans to support this idea. Humans who are mute and deaf or aphasic seem to have emotional experiences just like the rest of us, and there is little evidence that individual differences in any metacognitive ability are associated with individual differences in the ability to experience emotions. I realize that according to some psychological theories of emotion, there remains considerable room for debate here, but in my opinion, there is just no evidence at all to support the idea that the cognitive processes that dogs may lack, like language and metacognition, are necessary for experiencing emotions.

Given the completeness of emotional behaviors and brain functions that are being found in dogs, we should attribute subjective experiences of emotions to them. We are finding every single dependent measure that we find in humans, minus verbal report. The alternatives are to think of subjective experiences as somehow divorced from the web of the many functional interactions that are being discovered ("epiphenomenal" in some way), or as tied to very specific cognitive domains (language, metacognition). Neither view is compelling.

What remains is a realization of the difficulty in figuring out WHAT emotional experiences dogs have, but not WHETHER they have any at all. The names and concepts we have for our own experiences are unlikely to map well onto the experiences of dogs. Only careful work and the continuing accrual of more experimental data can allow us to assemble a new set of concepts for dog emotions. But the attribution of subjective experiences to accompany those emotions seems more justified than ever.

\section{References}

Berns, G. S., \& Cook, P. F. (2016). Why did the dog walk into the MRI? Current Directions in Psychological Science 25(5): 363-369.

Engle, R. W., \& Zentall, T. R. (2016). Editors' introduction: Special Issue on cognition in dogs. Current Directions in Psychological Science 25(5): 299.

Kujala, M. V. (2017). Canine emotions as seen through human social cognition. Animal Sentience 14(1). 